\title{
Dust Continuum Observations of Protostars: Constraining Properties with Simulations
}

\author{
Stella S. R. Offner
}

\begin{abstract}
The properties of unresolved protostars and their local environment (e.g., disk, envelope and outflow characteristics) are frequently inferred from spectral energy distributions (SEDs) through comparison with idealized model SEDs. However, if it is not possible to image a source and its environment directly, it is difficult to constrain and evaluate the accuracy of these derived properties. In this proceeding, I present a brief overview of the reliability of SED modeling by analyzing dust continuum synthetic observations of realistic simulations.
\end{abstract}

\section{Introduction}

Forming stars may be heavily obscured by their natal dust and gas, which inhibits direct imaging and causes source radiation to be reprocessed from shorter to longer wavelengths. The details of the multi-wavelength emission, i.e., the spectral energy distribution (SED), thus provide important indirect clues about the protostellar properties and environment. For example, the absence of $\leq 10 \mu \mathrm{m}$ emission usually signifies a very young source with a dense gas envelope; low or non-existent millimeter emission indicates a more evolved source, which has accreted or dispelled its envelope (e.g., [2]).

The information implicit in the reprocessed emission is commonly extracted by comparing the observed SED with idealized models of the protostellar source and gas distribution that are post-processed with a radiative transfer code to produce SEDs. Input models that reproduce the observed SED provide good candidate representations of the underlying source properties. These can provide a wealth of physical details (e.g., source mass, disk mass and radius, envelope density and radius, outflow cavity size, inclination) that would otherwise be impossible to obtain with

Stella S. R. Offner

Harvard-Smithsonian Center for Astrophysics, 60 Garden St. Cambridge, MA 02138, e-mail: soffnerecfa.harvard.edu 
observations. However, a number of caveats complicate this technique, including degeneracy between parameters, adoption of symmetry, and assumption that the observed SED represents a single source rather than a multiple system or even a small cluster [3].

Using simulations, which have completely known source and gas information, it is possible to assess the accuracy of this method applied to unresolved observed sources. In this proceeding, we summarize the results of [6], who present a comparison between the true properties of sources within simulations of a turbulent, star formatting cloud and the properties inferred from synthetic SEDs.

\section{Methods}

To perform the comparison, we follow three main steps (see Figure 11. First, we use the ORION adaptive mesh refinement (AMR) code to simulate a $0.65 \mathrm{pc}$ long turbulent cloud including self-gravity, radiation in the flux-limited diffusion approximation, and star particles inserted in regions of the flow exceeding the maximum resolution. The star particles are endowed with a sub-grid model for protostellar evolution and mass outflow launching based upon [4] (see [5] and [1] for implementation details). Both aspects are coupled to the instantaneous protostellar mass and accretion rate, which are modeled self-consistently through gas accretion from the AMR grid. For computational efficiency, we first perform the calculation with $200 \mathrm{AU}$ cell resolution and then "zoom" in to $4 \mathrm{AU}$ resolution by restarting and adding additional refinement at selected outputs.

Next, we "observe" the protostellar sources at each time output using Hyperion, a parallelized, Monte Carlo radiative transfer dust continuum code [7]. In each case, we observe from 20 different viewing angles in 10 apertures logarithmically spaced between 1,000 AU and 20,000 AU and at five different grid resolutions. Figure $1 \mathrm{~b}$ illustrates the SEDs of one source for four viewing angles observed with a $1000 \mathrm{AU}$ aperture.

We then compare the observed SEDs sampled at wavelengths appropriate for the 2MASS, Spitzer, and Bolocam instruments with the 200,000 model grid published by [8]. This study parametrized the input properties of the source, disk, envelope and outflow using 14 unique physical variables sampled over a wide range of apertures and 10 different viewing angles. This work also provides a simple means to identify models with good-fits to data and extract the corresponding parameter values. Here, we use these models to derive the properties for fits satisfying: $\chi^{2}-\chi_{\text {best }}^{2}<3 N$, where $N$ is the number of SED data points and $\chi_{\text {best }}$ is the best-fit model provided $\chi_{\text {best }}^{2}<30 N$. Finally, we compare four derived parameters with the true source properties. More complete comparisons are presented in [6]. 


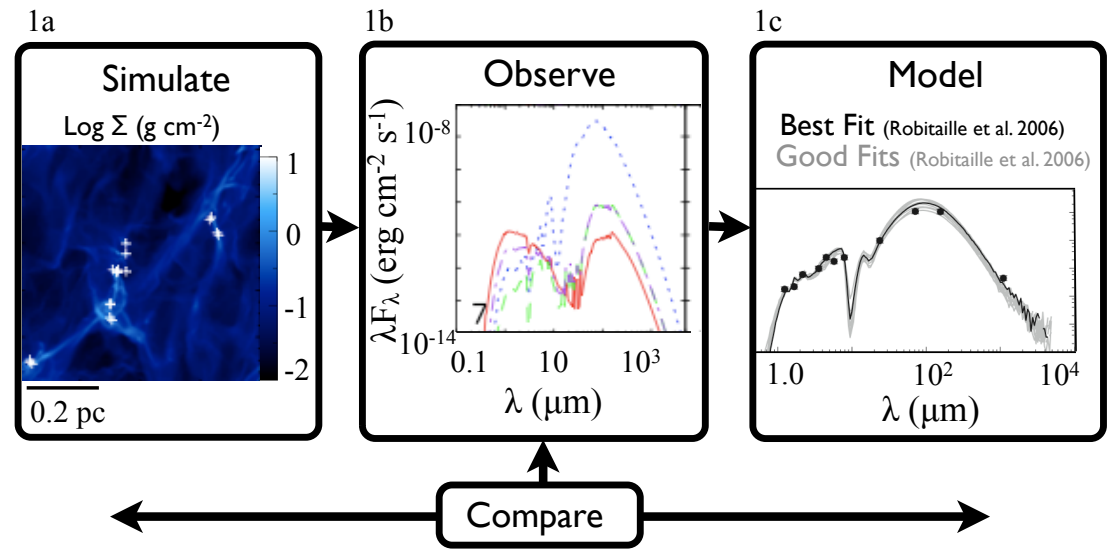

Fig. 1 Schematic of our process to assess the accuracy of properties inferred from SEDs. Panel 1a shows the log column density of the simulation $60 \mathrm{kyr}$ after the first star forms; protostar locations are marked with white crosses. Panel $1 \mathrm{~b}$ shows the SED of a source observed at four inclinations by the Hyperion code. Panel 1c shows a synthetic SED (black points) fit with SED models from the [8] model grid; the best-fit is indicated by the black line and good-fit cases are overlaid in gray.

\section{Results}

Figure 2 illustrates how well the good-fit models do in comparison to the simulated values for protostellar mass, protostellar radius, accretion rate, and source inclination. We find that the best-fit models give a reasonable estimate of the protostellar mass in the cases of more isolated or more massive protostars (generally those on the left half of Figure 21. This agreement occurs despite the discrepancy between the stellar evolution models, which causes the models from [8] to systematically overestimate the protostellar radius by a factor of 2-3. The origin of the discrepancy is the use of the model tracks by [9] that do not include accretion.

The range of inferred protostellar accretion rates typically encompass the true values, albeit with large spreads. The accretion rates of the good-fit models often extend over three to four orders of magnitude, which facilitates general agreement but highlights the difficulty of precisely constraining the true accretion rate. The source inclination proves to be a critical parameter in the comparison; in cases where the inclination is correctly well-constrained, the other inferred parameters tend to be more accurate. This suggests that placing limits on the inclination, e.g., via direct imaging, would improve the fidelity of the parameter estimation. 


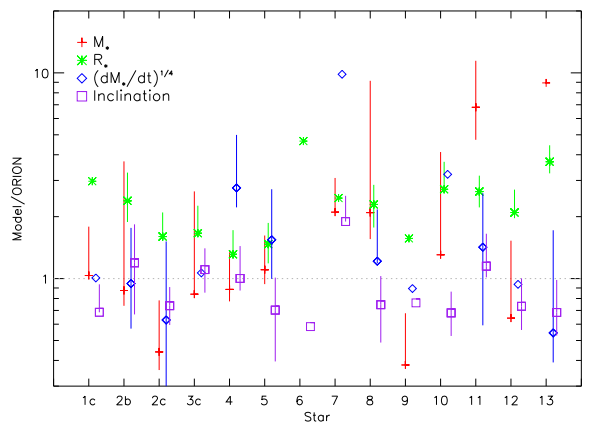

Fig. 2 Ratio of the inferred best model values to the actual simulation value for each source, where $M_{*}$ is the protostellar mass, $R_{*}$ is the protostellar radius, $\left(d M_{*} / d t\right)^{1 / 4}$ is the the accretion rate to the fourth power, and the inclination is the tilt with respect to the line of sight (reproduced from [6]). The dotted line indicates where the models correctly determine the simulation value.

\section{Conclusions}

Overall, the comparison between the simulations and analytic models underscores uncertainties inherent in modeling unresolved observations, especially in cases where the dust distribution, stellar evolution model, and gas geometry are not well constrained. Parameters inferred from the SEDs of unresolved sources should be accepted with caution and informed by direct imaging wherever possible.

Acknowledgements The author thanks the conference organizers for the opportunity to present this work and acknowledges Thomas Robitaille, Charles Hansen, Christopher McKee, and Richard Klein, who were collaborators in [6]. Figure 2 is reproduced by permission of the AAS. This work was supported by AST-0901055.

\section{References}

1. Cunningham, A. J., Klein, R. I., Krumholz, M. R., \& McKee, C. F. 2011, ApJ, 740, 107

2. Enoch, M. L., Evans, N. J., II, Sargent, A. I., \& Glenn, J. 2009, ApJ, 692, 973

3. Forbrich, J., Tappe, A., Robitaille, T., et al. 2010, ApJ, 716, 1453

4. Matzner, C. D. \& McKee, C. F. 1999 ApJ, 526, L109

5. Offner, S. S. R., Klein, R. I., McKee, C. F., \& Krumholz, M. R. 2009, ApJ, 703, 131

6. Offner, S. S. R., Robitaille, T. P., Hansen, C. E., McKee, C. F., \& Klein, R. I. 2012, ApJ, 753, 98

7. Robitaille, T. P. 2011 A\&A, 536, A79

8. Robitaille, T. P., Whitney, B. A., Indebetouw, R., Wood, K., \& Denzmore, P. 2006, ApJS, 167, 256

9. Siess, L., Dufour, E., \& Forestini, M. 2000, A\&A, 358, 593 